\title{
Über die Quantenbilanz photochemischer Prozesse und ihre Deutung nach der Treffertheorie
}

\author{
Von Karl H. Hausser \\ Aus dem Kaiser-Wilhelm-Institut für medizinische Forschung, \\ Abteilung für physikalische Therapie, Heidelberg \\ (Z. Naturforschg. 5 a, 41-55 [1950]; eingegangen am 1. August 1949)
}

Es wurden eine Reihe von Messungen über die Quantenbilanz photochemischer Wirkungen an organischen Molekülen ausgeführt, die folgende Resultate ergaben:

1. Die irreversible Umwandlung von Triphenyl-tetrazoliumchlorid (T.T.C., I) zu PhotoT.T.C (III) als Folge der Einstrahlung von ultraviolettem Licht ist nach Form der DosisEffekt-Kurve ein typischer Eintreffervorgang. Es wird jedoch etwa die vierfache Anzahl Quanten absorbiert, als bei einem Eintreffervorgang theoretisch zu erwarten ist, woraus sich der formale Treffbereich $v$ zu einem Viertel des Volumens $V$ der Einheit, des Moleküls, errechnet. Eine Abhängigkeit von der Wellenlänge oder von der Intensität des eingestrahlten Lichtes wurde innerhalb des untersuchten Bereiches nicht gefunden.

2. Die reversible cis-trans-Umwandlung von Triphenyl-formazan (T.F., II) in Benzol als Folge der Einstrahlung von sichtbarem Licht der Wellenlänge um $500 \mathrm{~m} \mu$ ist nach Form der Dosis-Effekt-Kurve ein typischer Eintreffervorgang. Die Anzahl der absorbierten Quanten ist jedoch im Mittel etwa 50-mal so groß wie die für einen Eintreffervorgang theoretisch berechnete Trefferzahl. Für diesen Vorgang wird eine Abhängigkeit des Ergebnisses von der Intensität des eingestrahlten Lichtes festgestellt.

3. Die irreversible Umwandlung von T.F. (II) zu Photo-T.T.C. (III) als Folge der Einstrahlung von ultraviolettem Licht ist nach Form der Dosis-Effekt-Kurve ein Mehrtreffervorgang. Die Resultate im einzelnen für die eingestrahlten Wellenlängen $\lambda=248,313$ und $366 \mathrm{~m} \mu$ zeigt Tab. 1. Durch Zusatz des bei 'T.T.C. in der Lösung vorhandenen Cl--Ions in Form von $\mathrm{HCl}$ wird die Anzahl der zur Umwandlung eines Moleküls notwendigen Treffer stark herabgesetzt; sie beträgt dann unabhängig von der eingestrahlten Wellenlänge etwa 4 Treffer. Die Größe des formalen Treffbereichs $v$ ist jedoch auch dann von der eingestrahlten Wellenlänge abhängig, sie beträgt bei $\lambda=313 \mathrm{~m} \mu$ etwa $1 / 110$, bei $\lambda=248 \mathrm{~m} \mu$ etwa $1 /{ }_{33}$. Zur Erklärung dieses Unterschiedes wird der Einfluß der mehr oder weniger günstigen Lage der Teile des Moleküls zueinander diskutiert.

Die experimentellen Ergebnisse unter 1, 2 und 3 können allgemein zusammengefaßt werden:

Bei der Beurteilung der Quantenbilanz photochemischer Prozesse muß man unterscheiden zwischen „absorbierten“ Quanten und den für einen bestimmten Prozeß zur Wirkung gelangenden „aktiven“ Quanten. Die Anzahl der pro Molekül „aktiv“ wirkenden Quanten läßt sich mit Hilfe der Treffertheorie ermitteln. Der Quotient aus der Anzahl der „aktiven“ und „absorbierten“ Quanten ergibt das Maß für den formalen Treffbereich, $\bar{q}_{\mathrm{akt}} / \bar{q}_{\mathrm{abs}}=v / \mathrm{V}$.

Durch Einsetzen der „aktiven“ an Stelle der „absorbierten“ Quanten wird eine neue Quantenempfindlichkeit $\gamma^{*}$ definiert als

$$
\gamma^{*}=\frac{\text { Anzahl der umgew. Molèküle }}{\text { Anzahl der aktiven Quanten }} \text {. }
$$

Es wird gezeigt, daß eine Aussage über die Quantenbilanz eines photochemischen Vorgangs dann vollständig ist, wenn außer der Quantenempfindlichkeit $\gamma$, bezogen auf die absorbierten Quanten, entweder der Quotient aus aktiven und absorbierten Quanten $\bar{q}_{\mathrm{akt}} / \bar{q}_{\mathrm{abs}}$ oder die neu definierte Quantenempfindlichkeit $\gamma^{*}$, bezogen auf die aktiven Quanten, angegeben wird.

\footnotetext{
Abeiten von I. Haußer, D. Jerchel, R. $\mathrm{Kuhn^{1,2 }}$ über photochemische Reaktionen von Triphenyl-formazan (T.F.) und Triphenyl-tetrazoliumchlorid (T.T.C.) hatten zu folgenden Resultaten geführt:

${ }^{1}$ I. Haußer, D. Jerchel u. R. Kuhn, Chem. Ber. 82, 195 [1949].
}

1. Bestrahlt man T.T.C. (I) oder T.F. (II) in Alkohol mit Licht ihrer UV-Absorptionsbande, $\lambda \approx 240$ bis $370 \mathrm{~m} \mu$, so wandeln sich beide irreversibel $\mathrm{zu}$ dem gleichen Photoprodukt um. Die Absorptionsspektren von T.T.C. (I) von T.F. (II), und von dem

${ }^{2} \mathrm{I}$. H a $\mathrm{H}$ er, D. J e rchel u. R. Kuhn, Chem. Ber. 82, 515 [1949]. 


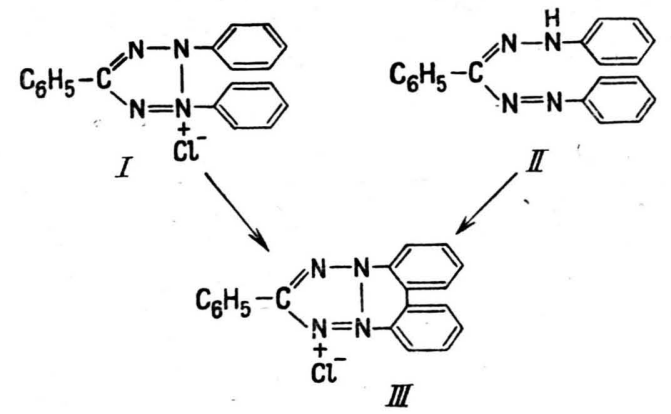

entstehenden Photoprodukt (III) zeigt Abb. 1. Das Photoprodukt konnte von D. Jerchel identifiziert werden als das Kation des 5-Phenyl-2.3-diphenylen-tetrazoliumchlorids (III) kurz Photo-T.T.C.

2. Bestrahlt man T.F. in Benzol mit Licht der langwelligen Absorptionsbande, $\lambda \approx 500 \mathrm{~m} \mu$, so ändert die Lösung ihre Farbe reversibel von rot in gelb, das Absorptionsspektrum geht dabei von Kurve a in Kurve b über (Abb. 2). Im Dunkeln tritt eine spontane Rückwandlung ein, deren Halbwertzeit in weiten Grenzen (bis zu einigen Stunden) mit dem $p_{\mathrm{H}}$-Wert schwankt - größere H-Ionen-Konzentration bedingt kürzere- Halbwertzeit.

In der vorliegenden Arbeit wird die Quantenbilanz der genannten photochemischen Vorgänge untersucht.

Bei der Messung der für die Umwandlung pro Molekül erforderlichen Quantenzahl ergab sich die Frage nach der statistischen Verteilung der absorbierten Quanten auf die absorbierenden Moleküle. Diese Verteilung gehorcht den Gesetzen der mathematischen Statistik. Die Beantwortung der Frage wurde daher durch die Anwendung dieser Gesetze auf die Meßergebnisse ermöglicht.

In der Biophysik hat sich die Anwendung mathematisch-statistischer Überlegungen auf biologische Strahlenwirkungen, die zuerst von F. D es s a u er angeregt und in der Folge von zahlreichen anderen zur Treffertheorie ausgebaut wurde ${ }^{3}$, als fruchtbar erwiesen und zur Klärung der Vorgänge bei der Absorption von Strahlungen in biologischen Objekten beigetragen. Die Anwendung der Treffertheorie wird jedoch in der Biologie dadurch erschwert, daß häufig Art und Größe der reagierenden Einheit und das Treffereignis selbst unbekannt sind. Ferner gibt die biologische Variabilität Veranlassung zur Mehrdeutigkeit der Ergebnisse.

Bei den vorliegenden photochemischen Reaktionen sind diese Unsicherheitsfaktoren nicht vorhanden.

${ }^{3}$ N. W. Timofé eff - R e s s ov s ky u. K. G. Z i m mer, Das Trefferprinzip in der Biologie. S. Hirzel, Stuttgart 1947 .
Das Treffereignis ist eindeutig definiert als die $\mathrm{Ab}$ sorption eines Lichtquants. Die reagierende Einheit ist das isolierte, in verdünnter Lösung befindliche, einzelne Molekül. Dadurch unterscheiden sich die

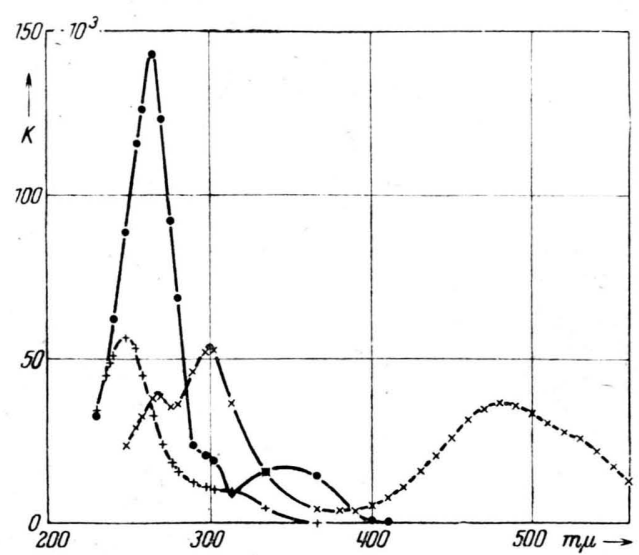

Abb. 1. Absorptionsspektren 2.3.5-Triphenyl-tetrazoliumchlorid (T.T.C.) -++ , Triphenyl-formazan(T.F.) $-\mathbf{x}-\mathbf{x}$ und 2.3-Diphenylen-5-phenyl-tetrazolium-chlorid (PhotoT.T.C.) - o-o in etwa 0,0001-m. alkoholischer Lösung. Ordinate: molarer Absorptions-Koeffizient $\varkappa$; AbszisseWellenlänge des Lichts in $\mathrm{m} \mu$.

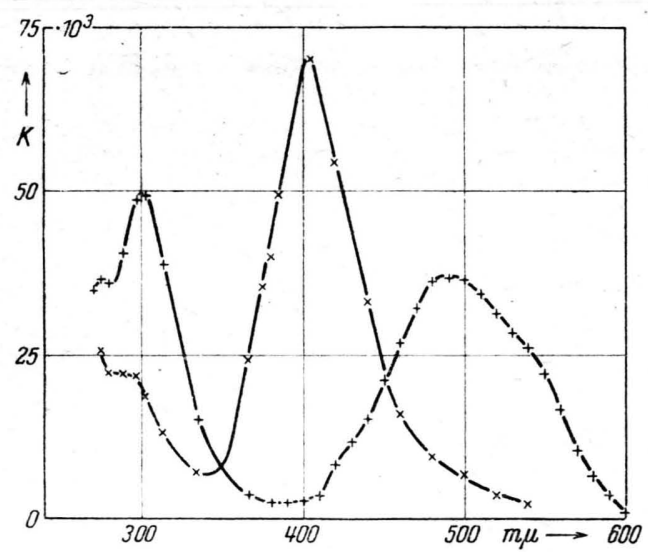

Abb. 2. Absorptionsspektrum von T.F. in etwa 0,0001- $m$. Benzol-Lösung. Lösung im Dunkeln angesetzt und gemessen: + - +- ; Lösung bei dauernder Belichtung mit sichtbarem Licht in kurzen Belichtungspausen gemessen $-x-x-x$ Ordinate: molarer Absorptions-Koeffizient $x$; Abszisse: Wellenlänge in mu.

hier untersuchten photochemischen Vorgänge auch von solchen, bei denen die reagierende Substanz in Form von Kristallgittern oder deren Bruchstücken vorliegt. Man kann daher erwarten, daß durch die Anwendung der Treffertheorie auf die hier behandelten photochemischen Reaktionen Einblicke in den Reaktionsmechanismus derselben ermöglicht werden. 


\section{Apparatur und Meßtechnik}

Die Absorptionsspektren wurden mit zwei Doppelmonochromatoren in der Anordnung nach R.W. $\mathrm{Pohl}^{4}$ aufgenommen, von denen der eine für das Sichtbare, der andere für das Ultraviolett (UV) verwendet wurde. Als Lichtquelle diente im Sichtbaren eine Wolframwendelfadenlampe $(8 \mathrm{~V}, 30 \mathrm{~W})$, im UV eine Quecksilberlampe. Die doppelte Zerlegung des Lichtes wurde im Sichtbaren durch Flintglasprismen, im UV durch Quarz- und Steinsalzprismen bewirkt. Die Messungen wurden durch lichtelektrische Photometrie an Lösungen gegen das jeweilige Lösungsmittel mit Hilfe von Hochvakuum-Alkali-Photozellen und Einfadenelektrometern durchgeführt. Zur Messung der Anzahl der absorbierten Quanten diente eine Anordnung, bei der durch einen lichtstarken Zeiß-Spektrographen konstanter Ablenkung $\left(90^{\circ}\right)$ spektral zerlegtes Licht nach Durchstrahlung der Meßküvette auf die lichtempfindliche Schicht einer Quarz-Hochvakuum-NatriumPhotozelle fiel. Lichtquelle war im sichtbaren Bereich eine Edelgas-Höchstdrucklampe $1000 \mathrm{~W}$ von Osram (HBO 1021) im UV eine ebensolche Quecksilber-Höchstdrucklampe (HBO 1001). Der durch den Photozellenstrom an einem Hochohmwiderstand von $5 \cdot 10^{9} \mathrm{Ohm}$ erzeugte Spannungsabfall wurde mit einem Einfadenelektrometer nach der Kompensationsmethode gemessen. Die spektrale Eichung der Photozelle im absoluten Energiemaß, die zur Bestimmung der Anzahl der Lichtquanten in den verschiedenen Spektralbereichen notwendig war, erfolgte durch Vergleich mit einem geeichten Vakuum-Thermoelement nach Prof. R. Hase.

Zur Kontrolle der Konstanz der Lichtquelle während der Umwandlung wurde ein Bruchteil des spektral zerlegten Lichtes mit Hilfe eines kleinen Quarzprismas unmittelbar vor der Küvette durch Totalreflexion abgezweigt und auf ein Selen-Photoelement eingestrahlt. Zur Messung des Photostromes der Selenzelle diente ein LichtmarkenGalvanometer von Siemens und Halske (Empfindlichkeit 6,6 $\cdot 10^{-8} \mathrm{~A} /$ Skt.), nach dessen Ausschlag die Lichtquelle nachgeregelt wurde.

\section{Voraussetzungen und theoretische Grundlagen}

Der Deutung der Meßergebnisse liegen die im folgenden ausgeführten Voraussetzungen und Überlegungen zu Grunde.

Zunächst seien die durch die Versuchsbedingungen gegebenen Voraussetzungen zusammengestellt:

1. In einer Küvette vom Volumen $U$, der Dicke $d$ und der Fläche $F_{\text {ges }}$ senkrecht zum Lichtstrom $\left(F_{\text {ges }}\right.$ $\boldsymbol{d}=U$ ) befinden sich bei Beginn des Versuchs $Z_{0}$ Moleküle im Zustand $\alpha$ in Lösung, $Z_{\alpha_{t=0}}=Z_{0}$.

2. In die Küvette wird ein zeitlich konstanter Lichtquantenstrom $J_{0}$ mit im ganzen Querschnitt gleicher Flächendichte eingestrahlt, von dem ein Teil $J$ von der Küvette hindurchgelassen wird, während ein anderer Teil $Q=J_{0}-J$ in derselben absorbiert wird.

' R. W. Po hl, Naturwiss. 15, 433 [1927].
3. Durch Absorption entweder a) eines Lichtquants oder, in anderen Fällen b) mehrerer Lichtquanten, geht ein Molekül des gelösten Stoffes vom Zustand $\alpha$ in den Zustand $\beta$ über.

4. Dié beiden Zustände $\alpha$ und $\beta$ sind durch die entsprechenden Absorptionsspektren der Lösung charakterisiert: bei mindestens einer Wellenlänge, der Meßwellenlänge $\lambda$, unterscheidet sich der molare Absorptions-Koeffizient $\varkappa_{a}$ des einen Zustandes um so viel von dem des anderen $\varkappa_{\beta}$, daß die kontinuierliche Änderung des gemessenen $x$-Wertes während der Umwandlung als Maß für den Prozentsatz der umgewandelten Moleküle dienen kann. (Die Proportionalität des gemessenen resultierenden $x$-Wertes mit der Änderung des Mischungsverhältnisses beider Komponenten wurde durch Messungen an Lösungen mit bekanntem Mischungsverhältnis kontrolliert.)

5. a) Bei der eingestrahlten Wellenlänge kann der für die theoretischen Überlegungen besonders einfache Spezialfall eintreten, daß für diese eine bestimmte Wellenlänge die Absorptions-Koeffizienten von Ausgangsmaterial und Endprodukt den gleichen Wert haben, also

$$
x_{\alpha, \text { eingestr. }}=x_{\beta} \text {, eingestr. }
$$

b) Im allgemeinen wird der Absorptions-Koeffizient des Photoprodukts für die eingestrahlte Wellenlänge einen anderen Wert haben als derjenige des Ausgangsmaterials, also

$$
\varkappa_{a, \text { eingestr. }} \neq \varkappa_{\beta} \text {, eingestr. }
$$

6. a) Es findet keine andere Reaktion statt außer der Umwandlung von Zustand $\alpha$ zum Zustand $\beta$ unter dem Einfluß der Lichtquanten; der Zustand $\beta$ ist stabil.

b) Es findet außer der Lichtumwandlung von $\alpha$ nach $\beta$ eine spontane Rückumwandlung von $\beta$ nach $\alpha$ statt; der Zustand $\beta$ ist instabil.

Bekanntlich ist bei einer gegebenen Wellenlänge $\lambda$ die durchgelassene Intensität $J$ mit der eingestrahlten $J_{0}$ verbunden durch die Gleichung

$$
J=J_{0} e^{-c d x}
$$

( $c=$ Konzentration der Lösung in $\mathrm{Mol} / l, d=$ Dicke der Küvette in $\mathrm{cm}$ ).

Demgemäß ist der molare Absorptions-Koeffizient $\varkappa$ definiert als:

$$
\varkappa=\frac{1}{c d} \ln \frac{J_{0}}{J} \text {. }
$$


Die Größe $\varkappa$ ist also, bei konstantem $c$ und $d$, ein Maß für das Verhältnis der Anzahl der in den gelösten Molekülen absorbierten Quanten zu den insgesamt eingestrahlten und damit ein Maß für den mittleren, bei der Absorption von Licht einer bestimmten Wellenlänge wirksamen Querschnitt der Moleküle. Bezeichnet man mit $F_{\text {ges }}$ den Querschnitt der Küvette senkrecht zum Lichtstrahl, so kann man denjenigen Bruchteil $F_{\mathrm{s}(\lambda)}$ davon, der für Quanten dieser Wellenlänge undurchlässig ist, durch die folgende Proportion definieren:

$$
F_{\mathrm{s}(\lambda)} / F_{\text {ges }}=\left(J_{0}-J\right) / J_{0} .
$$

Daraus läßt sich der für die Absorption wirksame Querschnitt $f_{(\lambda)}$ eines Moleküls in bezug auf Lichtquanten der Wellenlänge $\lambda$ berechnen zu:

$$
\begin{aligned}
f_{(\lambda)}= & \frac{F_{\mathrm{s}(\lambda)}}{Z_{0}} \\
& =\frac{10^{3}}{L d}\left(1-\frac{J}{J_{0}}\right)=\frac{10^{3}}{L c d}\left(1-e^{-\gamma_{c d}}\right)
\end{aligned}
$$

( $L=$ Loschmidtsche Zahl, $Z_{0}=$ Anzahl der Moleküle).

In der Biophysik besteht das bestrahlte Objekt aus einer bestimmten Anzahl sogenannter „biologischer Einheiten“ vom Volumen $V$. Als ,formaler Treff-

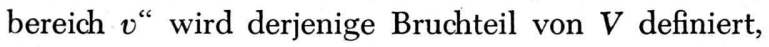
innerhalb dessen jede Absorption eines Lichtquants ein Treffer ist.

Nach der Treffertheorie kann man aus der Form der Dosis-Effektkurven auf die Anzahl der Treffer schließen, die zur Erzielung des Effektes notwendig sind. Dabei wird auf der Ordinate der Effekt in \% aufgetragen und auf der Abszisse der Quotient aus der mittleren Dosis pro Einheit und der Halbwertdosis, die zur Erzielung von 50\% Effekt notwendig ist. Besonders deutlich lassen sich mit Hilfe dieser Kurven Eintreffervorgänge von Mehrtreffervorgängen unterscheiden, da bei ersteren der Differentialquotient im Nullpunkt einen endlichen Wert hat, bei letzteren dagegen verschwindet.

Zur Anwendung der Treffertheorie auf die in dieser Arbeit vorliegenden Messungen der Lichtwirkung auf definierte organische Moleküle in Lösung werden im folgenden einige Definitionen angegeben. Ferner werden für die untersuchten reversiblen photochemischen Vorgänge einige Erweiterungen eingeführt, die

${ }^{5}$ Besondere Verhältnisse, wie sie z. B. bei Doppelmolekülen vorliegen, sollen hier zunächst unberücksichtigt bleiben. die spontane Rückbildung der bereits umgewandelten Moleküle rechnerisch zu berücksichtigen gestatten.

Der biologischen Einheit, also der reagierenden Einheit, entspricht hier im allgemeinen das gelöste organische Molekül ${ }^{5}$. Auf den Begriff des ,formalen Treffbereichs“ wird bei der Diskussion der experimentellen Ergebnisse näher eingegangen.

Im folgenden werden zunächst die Gleichungen für die einfacheren Eintreffervorgänge behandelt (Voraussetzung 3a) und dann die Mehrtreffervorgänge (Voraussetzung $3 \mathrm{~b}$ ).

$$
\text { Eintreffer-Vorgäng e }
$$

Es wird definiert: Die Anzahl der pro sec absorbierten Quanten $J_{0}-J=Q$. Ferner ist $q=Q / Z_{0}$ die Anzahl der zu einem Zeitpunkt im Mittel pro Molekül und sec absorbierten Quanten und schließlich

$$
\bar{q}=q t
$$

die Anzahl der bis zur Zeit $t$ insgesamt im Mittel pro Molekül absorbierten Quanten (die mittlere Dosis pro Einheit).

Die Untersuchungen dieser Arbeit wurden an Lösungen geringer Konzentration und kleiner Schichtdicke durchgeführt $(\varkappa c d<0,5)^{6}$. Unter diesen Voraussetzungen wird die Verteilung der absorbierten Lichtquanten auf die Moleküle in guter Annäherung durch das statistische Verteilungsgesetz von Poisson dargestellt. Danach ist die Wahrscheinlichkeit $w$ dafür, daß ein Molekül genau $n$ Treffer erhalten hat:

$$
w=\frac{q^{n} e^{-\bar{q}}}{n !} .
$$

Also beträgt die Anzahl $Z_{a}$ der Moleküle, die noch keinen Treffer bekommen haben und sich daher noch im Zustand $\alpha$ befinden:

$$
Z_{c}=Z_{0} e^{-q}
$$

und die Zahl derjenigen, die mindestens 1 Treffer erhalten haben und daher in den Zustand $\beta$ umgewandelt worden sind

$$
Z_{\beta}=Z_{0}\left(1-e^{-q}\right) .
$$

Bei zeitlich konstanter Einstrahlung und Absorption kann man die pro Molekül absorbierte Quanten-

${ }^{6}$ Eine Verallgemeinerung der Formel für die statistische Verteilung auf photochemische Prozesse in Lösungen großer Konzentration und Schichtdicke wird in einer gesonderten Mitteilung gegeben. 
summe $\bar{q}$ nach (5) ersetzen durch $q t$. Dadurch erhält man aus (7) und (8)

$$
\begin{aligned}
& Z_{a}=Z_{0} e^{-q t} \\
& Z_{\beta}=Z_{0}\left(1-e^{-q t}\right) .
\end{aligned}
$$

Die Differentiation der Gl. (9) nach $t$ ergibt

$$
\left(d Z_{a}\right)_{\text {Licht }}=-q Z_{a} d t .
$$

Der Index „Licht“ soll diese Änderung von $Z_{a}$ von derjenigen unterscheiden, die im Dunkeln spontan stattfindet. Der physikalische Inhalt von (11) ist unmittelbar deutlich: Die Abnahme der Zahl der Moleküle im Zustand $\alpha,\left(d Z_{c}\right)_{\text {Licht }}$ ist proportional der Zahl $Z_{\alpha}$ dieser Moleküle zur Zeit $t$, dem Zeitelement $d t$ und der Anzahl $q$ der pro Molekül und sec absorbierten Quanten, solange diese (nach Voraussetzung 5 a) eine Konstante ist.

Entsprechend ergibt die Differentiation von (10)

$$
\left(d Z_{\beta}\right)_{\text {Licht }}=q Z_{\alpha} d t .
$$

Für $t=0$ ist

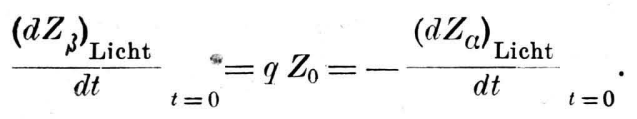

Die Ableitung ist also ungleich Null, wie für einen Eintreffervorgang zu erwarten.

Gilt nicht Voraussetzung $6 \mathrm{a}$, sondern $6 \mathrm{~b}$, d. h. findet gleichzeitig eine spontane Rückumwandlung statt, so liegen folgende physikalischen Verhältnisse vor: Die spontane Rückumwandlung ist der Anzahl der schon umgewandelten Moleküle $Z_{i \text { is }}$ proportional und außerdem einem Proportionalitätsfaktor $\mu$, der in einer nicht näher bekannten Weise vom $p_{\mathrm{H}}$ der Lösung und von der Temperatur, aber nicht von der Zeit $t$ abhängt. Man kann also für die im Dunkeln spontan erfolgende Abnahme - $\left(d Z_{i j}\right)_{\text {sp } p \text { nt. }}$ der Moleküle im Zustand $\beta$ bzw. die Zunahme $\left(d Z_{c}\right)_{\text {spont. }}$ der Moleküle im Zustand $\alpha$ den Ansatz machen

$$
-\left(d Z_{\beta}\right)_{\text {spont. }}=\left(d Z_{\alpha}\right)_{\text {spont. }}=\mu Z_{\beta} d t .
$$

Zusammen mit Gl.(11) erhält man für die gesamte Änderung $d Z_{a}$

$d Z_{\alpha}=\left(d Z_{c}\right)_{\text {Licht }}+\left(d Z_{\alpha}\right)_{\text {spont. }}=\left(-q Z_{\alpha}+\mu Z_{\beta}\right) d t$

und mit $Z_{\beta}=Z_{0}-Z_{\alpha}$

$$
d Z_{a}=\left[\mu Z_{0}-(\mu+q) Z_{a}\right] d t .
$$

Die Lösung dieser Differentialgleichung lautet mit der Anfangsbedingung $Z_{c, t=0}=Z_{0}$ :

$$
Z_{\alpha}=\frac{Z_{0}}{\mu+q}\left(\mu+q e^{-(\mu+q) t}\right) .
$$

Entsprechend ist die Änderung $d Z_{\beta}$

$$
d Z_{\beta}=\left(q Z_{a}-\mu Z_{\beta}\right) d t .
$$

Das ergibt mit der Anfangsbedingung $Z_{\beta},{ }_{t=0}=0$ :

$$
Z_{\beta}=\frac{q}{\mu+q} Z_{0}\left(1-e^{-(\mu+q) t}\right) .
$$

Der Endzustand für $t=\infty$ stellt ein Gleichgewicht dar, dessen Lage von dem Verhältnis von $\mu$ zu $q$ abhängig ist:

$$
Z_{j, t=\infty}=\frac{q}{\mu+q} Z_{0} .
$$

Die Ableitung im Nullpunkt ist die gleiche wie ohne spontane Rückumwandlung

$$
{\frac{d Z_{\beta}}{d t}}_{t=0}=q Z_{0} .
$$

Im Falle $5 \mathrm{~b}$, bei dem für die eingestrahlte Wellenlänge $\varkappa_{C} \neq x_{, 3}$ ist, ist nach der Voraussetzung

$$
J_{0}-J=Q=\varphi(t),
$$

d. h. die pro sec absorbierte Quantenzahl $Q$ der Lösung ist keine Konstante, sondern ändert sich während der Umwandlung als Funktion der Zeit. Folglich ist auch die pro Molekül und sec absorbierte Quantenzahl $q$ ebenfalls keine Konstante, sondern eine Funktion von $t$. Diese Änderung von $Q$ und $q$ mit der Zeit beruht auf der Änderung des Absorptionsquerschnittes bei der Umwandlung der Moleküle. Zur Ermittlung der Quantenausbeute sind aber nur die von nicht umgewandelten Molekülen $a b$ sorbierten Quanten von Bedeutung; deren $\mathrm{Ab}$ sorptionsquerschnitt und damit deren Absorptionskoeffizient $\varkappa_{\alpha}$ ist aber konstant $^{7}$. Man kann also im Anfangszustand, wenn sich alle Moleküle im Zustand $\alpha$ befinden, $x_{a}$ und damit $Q$ und $q$ bestimmen. Da die Änderung von $q$ nur auf der Änderung der Absorptionseigenschaften der umgewandelten Moleküle beruht, kann man für die Berechnung der Quantenausbeute die pro Molekül und sec absorbierte Quantenzahl $q$ als Konstante und zwar gleich $q_{0}$ an-

7 In diesem Zusammenhang sei auf die auf Seite 44 gemachte Voraussetzung geringer Konzentration und kleiner Schichtdicke nochmals besonders hingewiesen. 
setzen, sofern man nur dafür sorgt, daß die pro Zeitund Flächeneinheit eingestrahlte Quantenzahl $J_{0}$ konstant ist. Alle bisher entwickelten Formeln behalten damit auch für den Fall ihre Gültigkeit, daß bei der eingestrahlten Wellenlänge $\varkappa_{a}$ von $\varkappa_{\beta}$ verschieden ist.

Die eigentliche Meßgröße ist nicht $Z_{a}$ oder $Z_{\beta}$, sondern $\varkappa$. Da laut Voraussetzung 4 angenommen wurde, daß $x_{\text {gem }}$ sich bei einer geeigneten MeßWellenlänge in demselben Verhältnis wie die Anzahl $Z_{a}$ zu der Anzahl $Z_{\beta}$ ändert, ist $\varkappa_{\text {gem. }}$ also:

$$
\varkappa_{\text {gem }}=x_{a} \frac{Z_{a}}{Z_{0}}+x_{\beta} \frac{Z_{\beta}}{Z_{0}}
$$

oder mit $Z_{\beta}=Z_{0}-Z_{c}$

$$
x_{\text {gem }}=x_{\beta}+\left(x_{\alpha}-x_{\beta}\right) \frac{Z_{\alpha}}{Z_{0}} .
$$

Für den Fall ohne spontane Rückbildung ergibt sich also mit $Z_{a}=Z_{0} e^{-q t}$

$$
x_{\text {gem }}=x_{\beta}+\left(x_{c l}-x_{\beta}\right) e^{-q t} .
$$

Für den Fall mit spontaner Rückwandlung,

$$
\begin{gathered}
Z_{a}=\frac{Z_{0}}{\mu+q}\left(\mu+q e^{-(\mu+q) t}\right), \quad \text { erhält man } \\
x_{\text {gem }}=x_{\beta}+\frac{x_{\alpha}-x_{\beta}}{\mu+q}\left(\mu+q e^{-(\mu+q) t}\right) .
\end{gathered}
$$

\section{Mehrtreffer-Vorgänge}

Als Grundlage zur Auswertung der Meßresultate werden zunächst die Gleichungen für einen Zweitreffer-Vorgang aufgestellt, und zwar sowohl für den Fall reiner Umwandlung durch Licht (Voraussetzung 6a) als auch erweitert für den Fall gleichzeitiger spontaner Rückwandlung der Moleküle in der Lösung (Voraussetzung 6b).

Zur Diskussion der ebenfalls untersuchten Fälle von Vieltreffer-Vorgängen, die mathematisch nichts prinzipiell Neues bringen, deren Berechnung aber sehr langwierig ist, wird die von $\mathrm{Th}$ or $\mathrm{ndike} \mathrm{e}^{8} \mathrm{zu}$ diesem Zweck aufgestellte Tafel für das statistische Verteilungsgesetz nach Poisson benutzt.

Nach dem Poissonschen Verteilungsgesetz ist die Wahrscheinlichkeit für

$$
\begin{aligned}
& \text { genau } 0 \text { Treffer } w_{0}=e^{-\bar{q}}, \\
& \text { genau } 1 \text { Treffer } w_{1}=q e^{-q}, \\
& \text { höchstens } 1 \text { Treffer } v_{0,1}=e^{-q}(q+1),
\end{aligned}
$$

dementsprechend ist mit $\bar{q}=q t$ :

$$
\begin{gathered}
Z_{a}=Z_{0} e^{-q t}(q t+1), \\
Z_{\beta}=Z_{0}\left[1-e^{-q t}(q t+1)\right] .
\end{gathered}
$$

Die Differentiation der Gl. (26) ergibt

$$
d Z_{a}=-q Z_{a} \frac{q t}{q t+1} d t
$$

Der physikalische Inhalt dieser Differentialgleichung ist nicht so ohne weiteres einzusehen wie bei der entsprechenden für einen Eintreffer-Vorgang, doch ist einleuchtend, daß $d Z_{\alpha}$ nicht proportional $q$, sondern in komplizierter Form von $q$ und $t$ abhängig ist, wenn 2 oder mehr Treffer zur Erzielung eines Effekts erforderlich sind.

Für den Fall gleichzeitiger spontaner Rückwandlung erhält man mit $Z_{\beta}=Z_{0}-Z_{\ell}$ die erweiterte Differentialgleichung

$$
d Z a=\left[-\left(\mu+\frac{q^{2} t}{q t+1}\right) Z_{a}+\mu Z_{0}\right] d t
$$

die Lösung lautet mit der Anfangsbedingung

$$
\begin{aligned}
& Z_{\alpha t=0}=Z_{0}: \\
& Z_{\alpha}=Z_{0}(q t+1) e^{-(\mu+q) t} \\
& \cdot\left[1+\int_{0}^{t} \frac{e^{(\mu+q) x}}{q x+1} d x\right] .
\end{aligned}
$$

Das Integral läßt sich nicht elementar ausrechnen, aber auf eine tabellierte Funktion (Integral-Logarithmus) umformen. Dies geschieht durch dieSubstitution

$$
x=\frac{\ln s}{\mu+q}-\frac{1}{q} .
$$

Das Integral $\boldsymbol{J}$ geht dann über in

$$
\begin{aligned}
& J= \frac{e^{-\frac{\mu+q}{q}}}{q} \int_{e^{\frac{\mu+q}{q}}}^{e^{\frac{\mu+q}{q}}(q t+1)} \operatorname{ds} \\
& \ln s \\
&= \\
&=\frac{e^{-\frac{\mu+q}{q}}}{q}\left[\operatorname{li}\left(e^{\frac{\mu+q}{q}(q t+1)}\right)-\operatorname{li}\left(e^{\frac{\mu+q}{q}}\right)\right],
\end{aligned}
$$

wobei li $(x)=\int_{0}^{x} \frac{d t}{\ln t}, x>0$ den Integral-Logarith-

${ }^{8}$ F. Thorndike, Bell System Techn. J. 5, 604 [1926]. 
mus bezeichnet. Mit der Bezeichnung von Jahnke/Emde (Funktionentaf. S. 6) li $(x)=\overline{\mathrm{Ei}}[\ln (x)]$ wird

$J=\frac{e^{-\frac{\mu+q}{q}}}{q}\left[\overline{\mathrm{Ei}}\left\{\frac{\mu+q}{q}(q t+1)\right\}-\overline{\mathrm{Ei}}\left(\frac{u+q}{q}\right)\right]$,

dabei ist $\mu, q, t>0$.

Die Ableitung ist $\frac{d Z_{a}}{d t}{ }_{t=0}=0$ ebenso wie ohne spontane Rückbildung.

Betrachtet man wieder die $\varkappa$-Werte gemäß (22) so erhält man

$$
x_{\text {gem }}=x_{\beta}+\left(x_{\alpha}-x_{\beta}\right) e^{-q t}(1+q t) .
$$

Mit spontaner Rückbildung ergibt sich

$$
\begin{aligned}
& x_{\text {gem }}=x_{\beta}+\left(x_{\alpha}-x_{\beta}\right)(q t+1) e^{-(\mu+q) t} \\
& \cdot\left[1+\frac{\mu}{q} e^{-\frac{\mu+q}{q}}\left\{\overline{\operatorname{Ei}}\left(\frac{\mu+q}{q}(q t+1)\right)-\overline{\operatorname{Ei}}\left(\frac{\mu+q}{q}\right)\right\}\right] .
\end{aligned}
$$

\section{Experimentelle Ergebnisse und Diskussion}

Die Ergebnisse der Untersuchungen über die Quantenbilanz der photochemischen Umwandlung von T.T.C. (I) und T.F. (II) in Lösung durch Licht sind in den Kurven der Abb. 3 bis 15 zusammengestellt.

A. Für T.T.C.in alkoholischer Lösung zeigt Kurve 1 in Abb. 3 die gemessene Änderung des molaren Absorptionskoeffizienten $\varkappa$ bei der Meß-Wellenlänge $\lambda=265 \mathrm{~m} \mu$ als Folge der Einstrahlung von monochromatischem ultraviolettem Licht der Wellenlängen 313 oder $248 \mathrm{~m} \mu$. Während der Umwandlung wurde die pro sec eingestrahlte Quantenzahl $J_{0}$ konstant gehalten. Auf der Abszisse ist die im Mittel pro Molekül absorbierte Quantenzahl $\bar{q}=q_{0} t$ aufgetragen, auf der Ordinate der Absorptionskoeffizient $\varkappa$, der von dem für T.T.C. (I) charakteristischen Anfangswert $\varkappa_{I, 265}=30 \cdot 10^{3}$ in den für das Photoprodukt charakteristischen Wert $\varkappa_{I I I, 265}=120 \cdot 10^{3}$ übergeht. Der Wert des Absorptionskoeffizienten $\varkappa_{I I, 265} \approx$ $130 \cdot 10^{3}$ einer aus reinstem, isoliertem Photo-T.T.C. (III) hergestellten Lösung konnte durch Bestrahlung nicht ganz erreicht werden. Zur Berechnung des Prozentsatzes der Umwandlung wird im folgenden auf den höchsten durch Bestrahlung erzielbaren Wert von $\varkappa_{1 I I, 265}=120 \cdot 10^{3}$ bezogen. Die so erhaltene Umwandlungskurve in Prozenten als Funktion von $q$ zeigt Kurve 2 in Abb. 3. In Abb. 4 sind die Punkte

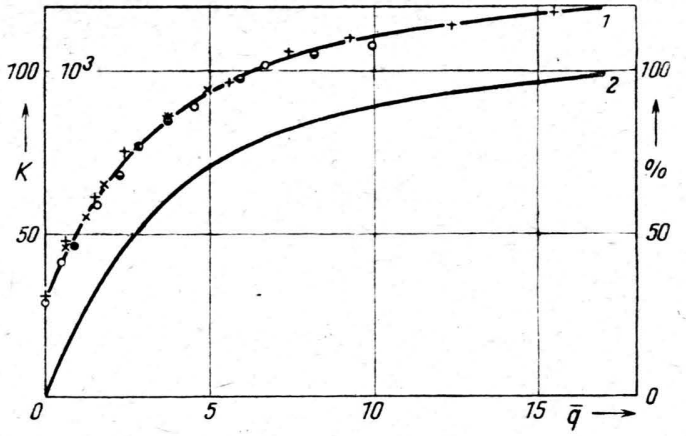

Abb. 3. Umwandlung von T.T.C. in alkohol. Lösung in das Photoprodukt durch ultraviolettes Licht. Ordinate: molarer Absorptions-Koeffizient $\varkappa$; Abszisse : mittlere absorbierte Quantenzahl pro Molekül $=\bar{q}$. Kurve 1: Änderung des molaren Absorptions-Koeffizienten $\varkappa$, gemessen bei $\lambda=265 \mathrm{~m} \mu$, hervorgerufen durch Einstrahlung von $\lambda=248 \mathrm{~m} \mu$ (0-0), durch Einstrahlung von $\lambda=313 \mathrm{~m} \mu$ bei verschiedenen Intensitäten,$++ \mathbf{x x} . \bullet \bullet$. Kurve 2: Umwandlung von T.T.C. zu dem Photoprodukt, in $\%$ berechnet aus Kurve 1.

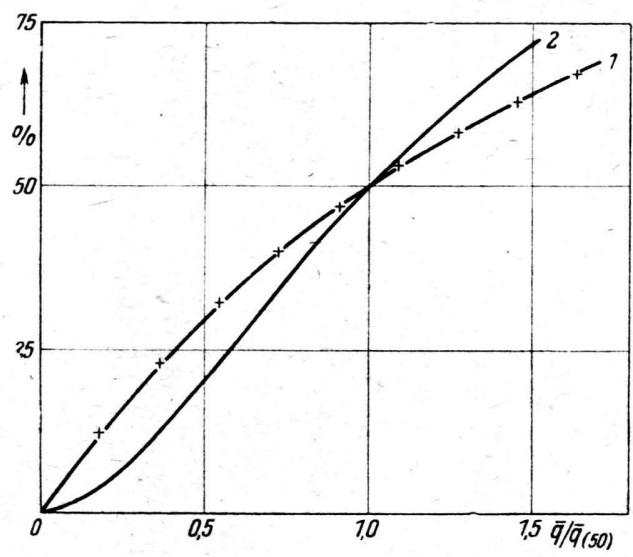

Abb. 4. Umwandlung von T.T.C. zu Photo-T.T.C, als Dosis-Effekt-Kurve. Ordinate: \% der Umwandlung; Abszisse: Quotient aus der mittleren pro Molekül absorbierten Quantenzahl $\bar{q}$ und der für die Umwandlung von $50 \%$ erforderlichen mittleren, pro Molekül absorbierten Quantenzahl $\bar{q}_{(50)}$. Kurve 1: theoretische EintrefferKurve; Kurve 2: theoretische Zweitreffer-Kurve. Aus den Messungen berechnete Dosis-Effekt-Kurve für die. Umwandlung von T.T.C.: +++ .

der daraus berechneten Dosis-Effekt-Kurve eingetragen. Zum Vergleich sind ferner die theoretischen Eintreffer- und Zweitrefferkurven als ausgezogene Kurven 1 und 2 eingezeichnet. Die aus den Messungen sich ergebenden Punkte fallen in guter Annäherung mit der theoretischen Eintrefferkurve zusammen. Auf Grund der Form der Dosis-EffektKurve ergibt sich also die Umwandlung von T.T.C. (I) in Photo-T.T.C. (III) als Eintreffer-Vorgang. 
Bei einem Eintreffer-Vorgang sind nach der Treffertheorie 50\% Umwandlung bei einem Wert von im Mittel 0,7 Treffer pro Einheit zu erwarten. Kurve 2 in Abb. 3 erreicht jedoch 50\% Umwandlung erst bei 2,8 im Mittel pro Molekül absorbierten Quanten. Daraus geht hervor, daß man keineswegs „,absorbiertes Quant“ und „Treffer" gleichsetzen kann. Im vorliegenden Fall z. B. ist nur etwa jedes 4. absorbierte Quant ein Treffer. Man muß daher dem Begriff des ,absorbierten“ Quants denjenigen des bei einem speziell betrachteten Vorgang als Treffer zur Wirkung gelangenden, ,aktiven“ Quants gegenüberstellen; es ist zu unterscheiden zwischen der Anzahl der absorbierten Quanten $\bar{q}_{\text {abs }}{ }^{9}$ und derjenigen der aktiven Quanten $\bar{q}_{\text {akt }}$ Zur vollständigen Aussage über die Quantenbilanz eines photochemischen Vorgangs gehört demnach

1. Die Bestimmung der Anzahl der bis zur Erreichung eines bestimmten Prozentsatzes der Umwandlung (z. B. 50\%) im Mittel absorbierten Quanten $\bar{q}_{\mathrm{abs}, 50}$.

2. Die Bestimmung der zum Hervorrufen des Effekts im Molekül erforderliche Trefferzahl $n$ aus der Dosis-Effekt-Kurve.

3. Die Bestimmung der Anzahl der bis zur Erreichung des gleichen Prozentsatzes der Umwandlung wie bei 1 . aktiv wirkenden Quanten $\bar{q}_{\mathrm{akt}, 5 n}$ aus der Trefferzahl $n$ mit Hilfe des statistischen Verteilungsgesetzes von Poisson.

Der Quotient aus der Anzahl der aktiven Quanten $\bar{q}_{\mathrm{akt}}$ und derjenigen der absorbierten Quanten $\bar{q}_{\mathrm{abs}}$ entspricht dem in der Treffertheorie definierten „formalen Treffbereich $v$ " als Bruchteil des Volumens $V$ der reagierenden Einheit.

In dem hier untersuchten Beispiel beträgt

$$
\begin{gathered}
q_{\mathrm{abs}, 50}=2,8, \quad n=1, \quad \bar{q}_{\mathrm{akt}, 50}=0,7, \\
\frac{v}{V}=\frac{\bar{q}_{\mathrm{akt}, 50}}{\bar{q}_{\mathrm{abs}, 50}}=\frac{1}{4} .
\end{gathered}
$$

Ein Einfluß der Änderung der eingestrahlten Wellenlänge $(\lambda=248$ bzw. $313 \mathrm{~m} \mu)$ auf dieses Ergebnis konnte nicht festgestellt werden. Eine Änderung der Intensität $J_{0}$ des eingestrahlten Lichtes im Verhältnis 1:8 ergab ferner die Unabhängigkeit des Resultats von Intensitätsänderungen.

$\begin{array}{lll}9 & \bar{q} \text { bedeutet allein schon laut Definition die im Mittel }\end{array}$ pro Molekül absorbierte Quantenzahl, der Index „abs“ ist nur zur besseren Unterscheidung von der Anzahl der aktiven Quanten $\bar{q}_{\text {akt }}$ hinzuzufügen.
Es sei besonders darauf hingewiesen, daß der formale Treffbereich $v$ nur eine formale Rechengröße darstellt; seine Bedeutung liegt darin, daß er aus den experimentellen Daten berechnet werden kann. Die physikalisch realen Größen sind der wahre Treffbereich $\tau$ und die Wirkungswahrscheinlichkeit $p$, die mit dem formalen Treffbereich $v$ verbunden sind durch die Beziehung

$$
v=p \tau .
$$

Von den unendlich vielen Möglichkeiten, nach denen sich $v$ aus $p$ und $\tau$ zusammensetzen kann, seien die beiden Grenzfälle an dem Beispiel der Umwandlung von T.T.C. diskutiert. Aus den Versuchsergebnissen errechnet sich der formale Treffbereich $v$

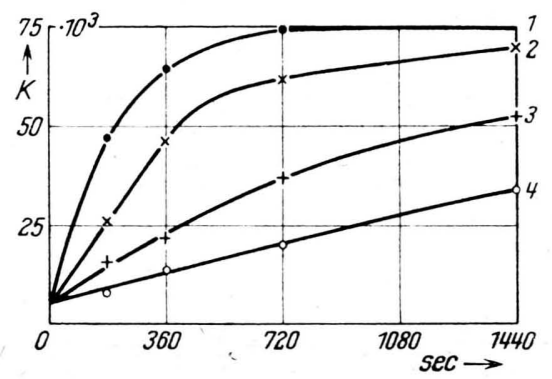

Abb. 5. Umwandlung von T.F. in Benzol durch sichtbares Licht, $\lambda=$ etwa $500 \mathrm{~m} \mu$, durch cis-trans-Umlagerung aus der roten in die gelbe Form. 4 Intensitätsstufen des eingestrahlten Lichts. Kurven $1: 2: 3: 4=J_{0}: J_{0} / 2: J_{0} / 4: J_{0} / 8$. Ordinate molarer Absorptions-Koeffizient $\varkappa$; Abszisse: Zeit in sec.

zu 1/4 des Volumens der Einheit, des Moleküls. Man kann nun einerseits annehmen, daß der wahre Treffbereich $\tau$, das reale Volumen, gleich dem Volumen des Moleküls ist, dann folgt für die Wirkungswahrscheinlichkeit $p=1 / 4$. Man kann aber auch die Wirkungswahrscheinlichkeit gleich 1 setzen, dann beträgt das Volumen des wahren Treffbereichs 1/4 des Volumens der Einheit. Zwischen diesen Möglichkeiten kann nicht mit Hilfe der Treffertheorie, sondern nur mit anderen physikalischen oder chemischen Überlegungen entschieden werden. Die zweite Möglichkeit, $\tau=v / 4, p=1$ kann bedeuten, daß die Energie des Lichtquants zur Erzielung eines Effekts an einer bestimmten, lokalisierten Stelle im Molekül zur Wirkung gelangen muß. Die Wahrscheinlichkeit $p=1 / 4$ im ersteren Fall dagegen kann man sich z. B. dadurch entstanden denken, daß die einzelnen Teile des Moleküls im Moment der Absorption eine für das Eintreten der Reaktion günstige, bestimmte Lage zueinander haben müssen, die infolge der mehr oder 
weniger eingeschränkten freien Drehbarkeit einzelner Atomgruppen im Molekül nicht immer gegeben ist.

B. Die Ergebnisse des durch die Absorptionsspektren (Abb. 2) charakterisierten photochemischen Effekts an T.F. (II) in Benzol-Lösung sind in den Abb. 5-8 dargestellt. Abb. 5 zeigt die gemessene Änderung des Absorptionskoeffizienten $\varkappa_{405}$ als Funktion der Zeit bei Bestrahlung der Lösung mit monochromatisch sichtbarem Licht zeitlich konstanter Intensität der Wellenlänge $\lambda \approx 500 \mathrm{~m} \mu$. Die Kurven 1, 2, 3, 4 unterscheiden sich dadurch, daß bei ihnen die pro sec und Molekül absorbierte Quantenzahl $q$ im Verhältnis etwa $1: 1 / 2: 1 / 4: 1 / 8$ abnimmt. Die Wirkung besteht in diesem Falle in einem Farbumschlag von rot nach gelb, der auf dem Umklappen einer Doppelbindung von der cis- in die trans-Form beruht. Welche der möglichen cis-trans-Kombinationen der roten bzw. der gelben Lösung zuzuordnen sind und mit welcher Berechtigung von definierten cis-trans-Isomerien gesprochen werden kann, ist an anderer Stelle ${ }^{2}$ diskutiert worden.

Als Meß-Wellenlänge wurde die Wellenlänge $\hat{\lambda}=405 \mathrm{~m} \mu$ benutzt, bei der der Absorptionskoeffizient $\varkappa$ von $5 \cdot 10^{3}$ (rote Lösung) auf $75 \cdot 10^{3}$ (gelbe Lösung) ansteigt.

Bei der Auswertung der Meßergebnisse ist zu beachten, daß der Farbumschlag, also die Wirkung, in diesem Fall reversibel ist. Im Dunkeln tritt spontane Rückumwandlung ein, deren Halbwertzeit außer von der Temperatur in weiten Grenzen vom $p_{\mathrm{H}}$ der Lösung abhängt. Die Berechnung der Zahl der umgewandelten Moleküle, also des Prozentsatzes der Umwandlung, muß daher nach den für die Voraussetzung $6 \mathrm{~b}$ entwịckelten Gln. (24) und (34) erfolgen, in denen die spontane Rückumwandlung berücksichtigt ist.

Bei den untersuchten Lösungen war die Halbwertzeit der spontanen Rüekumwandlung groß gegen die zur 50 -proz. Lichtumwandlung erforderliche Zeit, so daß sich für den Verlauf der Kurven in Abb. 5 bis 8 auf Grund der spontanen Rückumwandlung nur relativ geringfügige Korrektionen ergeben, die desto kleiner sind, je größer die Lichtintensität und je kürzer damit die Umwandlungszeit ist.

Da die gelbe Form von T.F. (II) bisher nur in Lösung unter Einstrahlung von Licht realisierbar ist, wurde für die Berechnung des Prozentsatzes der Umwandlung der höchste, bei stärkster Lichtintensität erreichbare Wert von $\varkappa_{405}=75 \cdot 10^{3}$ zugrunde gelegt. Die so erhaltenen Umwandlungskurven in \% als Funktion der Zeit sind als Kurven $1-4$ in Abb. 6

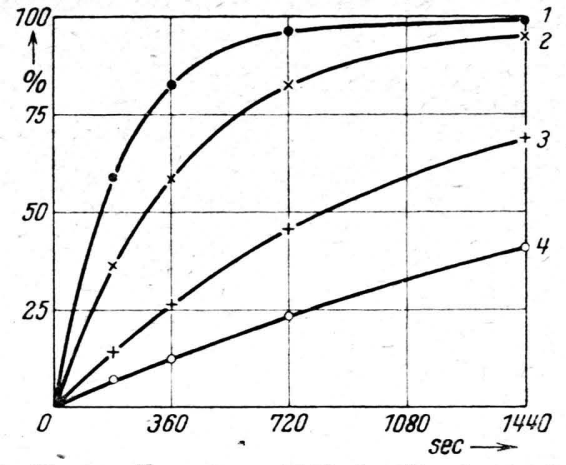

Abb. 6. Umwandlung von T.F. in $\%$ als Funktion der Zeit, berechnet aus Abb. 5. 4 Intensitätsstufen des eingestrahlten Lichts. Kurven $1: 2: 3: 4=J_{0}: J_{0} / 2: J_{0} / 4: J_{0} / 8$.

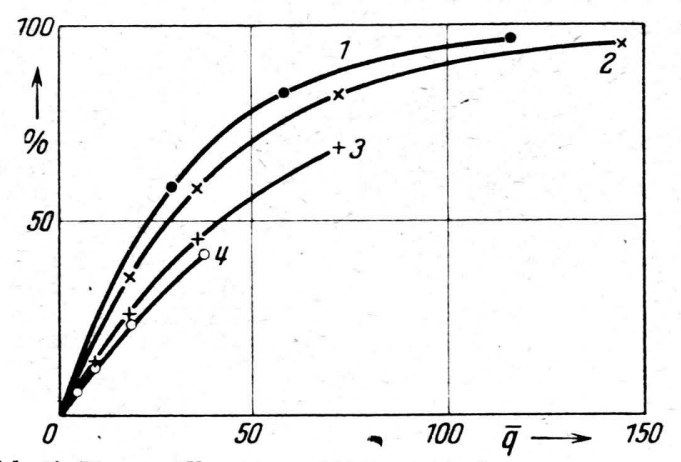

Abb. 7. Umwandlung von T.F. in \% als Funktion der im Mittel pro Molekül absorbierten Quantenzahl $\bar{q}$. 4 Intensitätsstufen des eingestrahlten Lichts. Kurven $1: 2: 3: 4$ $=J_{0}: J_{0} / 2: J_{0} / 4: J_{0} / 8$.

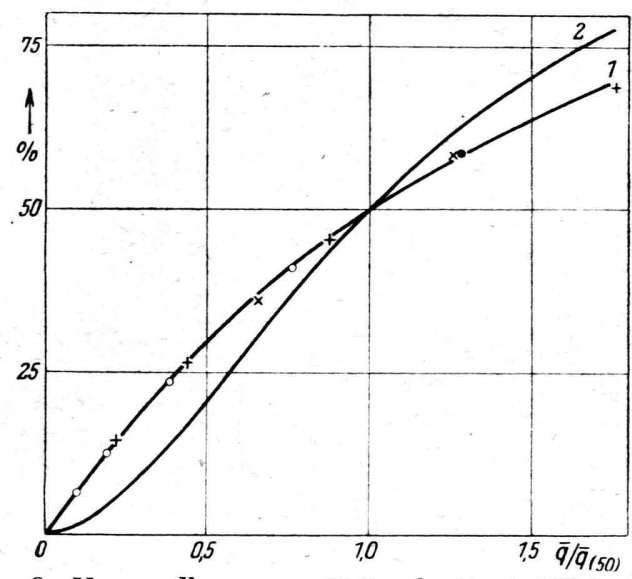

Abb. 8. Umwandlung von T.F. als Dosis-Effekt-Kurve. 1 und 2: Theoretische Ein- bzw. Zweitreffer-Kurve, berechnet unter Berücksichtigung einer mittleren spontanen Rückumwandlung. Aus den Messungen berechnete DosisEffekt-Kurve für die Rot-Gelb-Umwandlung von T.F.: $+-+, x-x, 0-0, v-v$. Ordinate: Umwandlung aus der roten in die gelbe Form in \%. Abszisse: Quotient aus der mittleren, pro Molekül absorbierten Quantenzahl $\bar{q}$ und der für die Umwandlung von $50 \%$ erforderlichen mitt-

leren, pro Molekül absorbierten Quantenzahl $\bar{q}_{(50)}$. 
dargestellt. Abb. 7 zeigt die gleichen Kurven als Funktion der pro Molekül im Mittel absorbierten Quantenzahl $\bar{q}$. In Abb. 8 sind als ausgezogene Kurven die mit Berücksichtigung der spontanen Rückumwandlung unter Annahme einer mittleren Lichtintensität theoretisch berechneten Eintreffer- und Zweitrefferkurven aufgetragen. Außerdem sind die Kurven 1-4 der Abb. 7 auf Dosis-Effekt-Kurven umgerechnet und in Abb. 8 als Punkte eingezeichnet.

Aus der guten Übereinstimmung aller aus den Versuchsergebnissen der Kurven 1-4 errechneten Werte mit der theoretischen Eintrefferkurve geht hervor, daß auch die cis-trans-Umwandlung von T.F. ein Eintreffervorgang ist. Es ist jedoch auch hierbei keineswegs ,,absorbiertes Quant“ und „Treffer“ identisch, sondern es ergibt sich für das Verhältnis $\bar{q}_{\mathrm{akt}}$. $\vec{q}_{\text {abs }}$ im Mittel ungefähr 1/50. Vom Standpunkt der Quantenbilanz stimmt dieses Resultat seinem Charakter nach mit dem bei der Umwandlung von T.T.C. zu Photo-T.T.C. gefundenen überein, wobei es für diese zunächst mathematisch-formalen Überlegungen ohne Einfluß ist, daß es sich chemisch um zwei ganz verschiedene Vorgänge handelt. Die oben angestellten Überlegungen über den realen Treffbereich $\tau$ und die Wirkungswahrscheinlichkeit $p$ behalten daher auch für die cis-trans-Umwandlung von T.F. ihre Gültigkeit.

Die Meßresultate der rot-gelb-Umwandlung von T.F. weichen jedoch in einem grundsätzlichen Punkt von den bei der Umwandlung von T.T.C. erhaltenen ab. Für die Kurven 1-4 der Abb. 7, die sich durch die prosec und Molekül im Mittel absorbierte Quantenzahl $q$ unterscheiden,ist der Quotient $\bar{q}_{\mathrm{Akt}} / \bar{q}_{\mathrm{ahs}}$ verschieden. Die Kurve 1, die der höchsten Intensitätsstufe entspricht, erreicht $50 \%$ Umwandlung bei etwa $23 \mathrm{im}$ Mittel pro Molekül absorbierten Quanten, während bei der geringsten Intensitätsstufe, Kurve 4, derselbe Umwandlungsprozentsatz erst bei etwa 50 im Mittel pro Molekül absorbierten Quanten erzielt wird.

Prinzipiell könnte diese Intensitätsabhängigkeit der Anzahl der bis zu einem bestimmten Umwandlungsprozentsatz absorbierten Quanten auf der spontanen Rückumwandlung von gelb nach rot beruhen. Denn infolge der Gegenläufigkeit von Lichtumwandlung und spontaner Rückumwandlung ist die Steilheit des Anstiegs der resultierenden Umwandlungskurve von dem Verhältnis der Geschwindigkeiten der beiden Einzelreaktionen abhängig, also von dem Verhältnis von $q \mathrm{zu} \mu$. Bei einem Vorgang mit spontaner Rückumwandlung ist keine hundertprozentige Umwand- lung möglich; der bei unendlicher Bestrahlungszeit theoretisch erreichbare höchste Prozentsatz der Umwandlung ist ebenfalls von dem Verhältnis von $q$ zu $\mu$ abhängig.

Quantitativ ist jedoch bei den gegebenen Versuchsbedingungen - die Halbwertzeit der spontanen Rückumwandlung war groß gegen diejenige der Lichtumwandlung, $\mu \ll q-$ die spontane Rückumwandlung von gelb nach rot keinesfalls ausreichend zur Erklärung der Steilheitsunterschiede der Kurven 1-4 der Abb. 7.

Nach den Arbeiten von R. Kuhn und D. Jer$\mathrm{chel}^{10}$ sowie L. Hunter und C. B. Roberts ${ }^{11}$ gehört das H-Atom im T.F. nicht einem von den beiden den Phenylringen benachbarten N-Atomen an, , sondern durch Wasserstoffbrückenbildung beiden zugleich, so daß Nebenvalenz-Ringbildung vorliegt. Es besteht die Möglichkeit, daß zwischen der Existenz der Wasserstoffbrücke und der gemessenen Intensitätsabhängigkeit ein Zusammenhang vorhanden ist. Eine endgültige Deutung dieses Ergebnisses søll bis zum Vorliegen weiteren experimentellen Materials zurückgestellt werden.

C. Die Quantenbilanz der Umwandlung von T.F. (II) zu Photo-T.T.C. (III) in alkoholischer Lösung zeigen die Abb.9-12. Die gemessene Änderung des Absorptionskoeffizienten bei den Meß-Wellenlängen $\lambda_{1}=490 \mathrm{~m} \mu$ und $\lambda_{2}=265 \mathrm{~m} \mu$ ist als Funktion der im Mittel pro Molekül absorbierten Quantenzahl $\bar{q}$ in der Abb. 9 dargestellt. Die Änderung von $\varkappa$ wurde durch Einstrahlung von monochromatischem Licht der Wellenlänge $\lambda=313 \mathrm{~m} \mu$ hervorgerufen. Gesetzmäßige Unterschiede im Verlauf dieser Kurven mit der Änderung der pro sec und Molekül absorbierten Quantenzahl $q$ konnten nicht sichergestellt werden.

Bei der UV-Bestrahlung von T.F. in alkoholischer Lösung manifestiert sich die Bestrahlungswirkung. die in der Umwandlung des Moleküls von T.F. (II) in das Photoprodukt (III) besteht, in zwei Erscheinungsformen. Es darf nicht ohne weiteres vorausgesetzt werden, daß sich beide Erscheinungsformen mit dieser irreversiblen Umwandlung von T.F. dekken. Beobachtet wird erstens, daß die ursprünglich rote Lösung vollkommen entfärbt wird, was dem $\mathrm{Ab}$ sinken des Absorptionskoeffizienten $\varkappa_{490}$ vom Anfangswert $\varkappa_{\text {II } 490}=36 \cdot 10^{3}$ auf Null entspricht (s. die unteren Kurven b in der Abb. 9). Zweitens ändert

${ }_{10}^{10}$ R. Kuhn u. D. J e r chel, Ber. dtsch. chem. Ges. 74, 941 [1941].

${ }_{11}^{11}$ L. Hunter u. C. B. Roberts, J. chem. Soc. [London] 1941, 820. 
sich das Absorptionsspektrum im UV, was sich durch den Anstieg von $\varkappa_{265}$ von dem Anfangswert $\varkappa_{\text {II, } 265}$ $=41 \cdot 10^{3}$ auf den Wert für das Photoprodukt (III) $\varkappa_{\text {III, }{ }_{265}}=120 \cdot 10^{3}$ anzeigt (s. die oberen Kurven a in der Abb. 9).

Die aus den Meßresultaten der Abb. 9 errechneten Umwandlungskurven in \% als Funktion der mittleren pro Molekül absorbierten Quantenzahl $\bar{q}$ stellt die Abb. 10 dar. Die Umwandlungskurve, die sich durch den Anstieg von $\varkappa_{265}$ anzeigt, ist in der Abb. 10 als Kurve a wiedergegeben, während die aus der Rotentfärbung berechnete als Kurve $b$ dargestellt ist.

Bei der Berechnung der prozentualen Umwandlung aus der Rotentfärbung war ein Störeffekt zu berücksichtigen. $\mathrm{Zu}$ dieser Berechnung wird als Bezugspunkt der Wert von $\varkappa_{0}$ bei $\mathrm{t}=0$ gebraucht. Dieser Wert ist kleinen Schwankungen unterworfen (etwa 5\%), die trotz zahlreicher Bemühungen nicht zuverlässig eliminiert werden konnten. Auch geht $\varkappa$ mit einem stufenförmigen Verlauf von dem gemessenen Anfangswert $\varkappa_{0}$ bei $t=0$ in dieS-förmige Kurve über, die den zeitlichen Ablauf der Entfärbung darstellt. Aus anderen, hier nicht berührten Beobachtungen ist anzunehmen, daß die beobachteten kleinen Schwankungen von $\varkappa_{0}$ (bei $\lambda=490 \mathrm{~m} \mu$ ) dem Umklappen der $\mathrm{N}=\mathrm{N}$-Doppelbindung zuzuschreiben sind. Der Übergang der $\mathrm{N}=\mathrm{N}$-Doppelbindung aus der cis- in die trans-Form erfolgt offenbar mit sehr geringem Quantenbedarf und ruft nur eine kleine Änderung von $\%$ hervor. Da das Umklappen der $\mathrm{N}=\mathrm{N}$-Doppelbindung nicht unmittelbar zu dem hier gemessęnen Vorgang der irreversiblen Entfärbung der T.F.-Lösung gehört, wurde die für den untersuchten Entfärbungsvorgang charakteristische S-Kurve bis zur Abszisse $t=0$ verlängert (in der Abb. 9 gestrichelt). Der Schnittpunkt der Verlängerung mit der Ordinate im Nullpunkt wurde als Ausgangspunkt $x_{0}$ für die Berechnung des Prozentsatzes der Umwandlung verwendet.

Die aus den Kurven der Abb. 10 errechneten DosisEffekt-Kurven der Umwandlung von T.F. sind in den Abb. 11 und 12 ausgezogen. Die Umwandlung wurde auch durch Einstrahlung der Wellenlänge $\lambda=248 \mathrm{~m} \mu$ und $\lambda=366 \mathrm{~m} \mu$ hervorgerufen. Die bei diesen Wellenlängen gemessenen Umwandlungskurven bringen gegenüber den Abb. 9 und 10 nichts prinzipiell Verschiedenes; die für diese eingestrahlten Wellenlängen errechneten Dosis-Effekt-Kurven sind in Abb. 11 und 12 ebenfalls als ausgezogene Kurven eingetragen. Abb. 11 zeigt die Dosis-Effekt-Kurven,

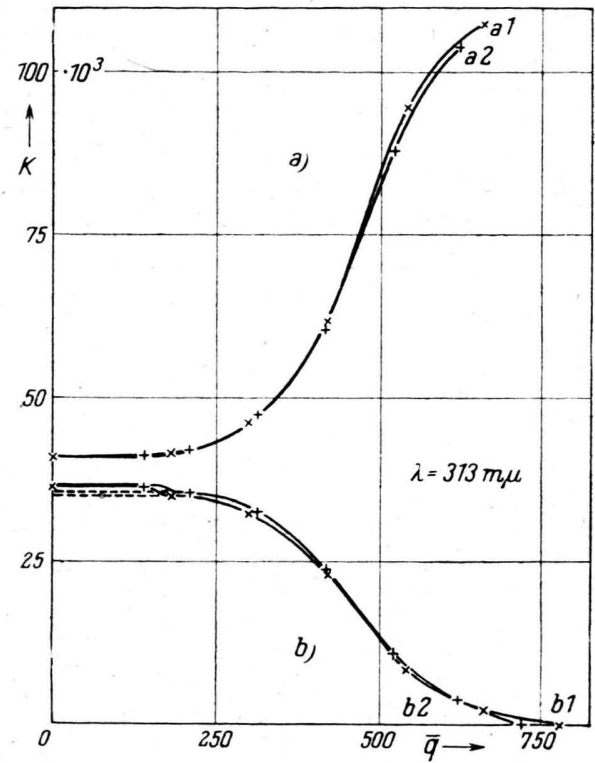

Abb. 9. Änderung des Absorptionskoeffizienten $\varkappa$ von T.F. in Alkohol durch Absorption von Licht der Wellenlänge $\lambda=313 \mathrm{~m} \mu$, zwei Meßreihen (Kurven 1 und 2) bei jeder Meßwellenlänge; Kurven a: $\lambda=265 \mathrm{~m} \mu$; Kurven $\mathrm{b}: \lambda=490 \mathrm{~m} \mu$. Abszisse: mittlere absorbierte Quantenzahl pro Molekül $\bar{q}$; Ordinate: molarer Absorptionskoeffizient $x$.

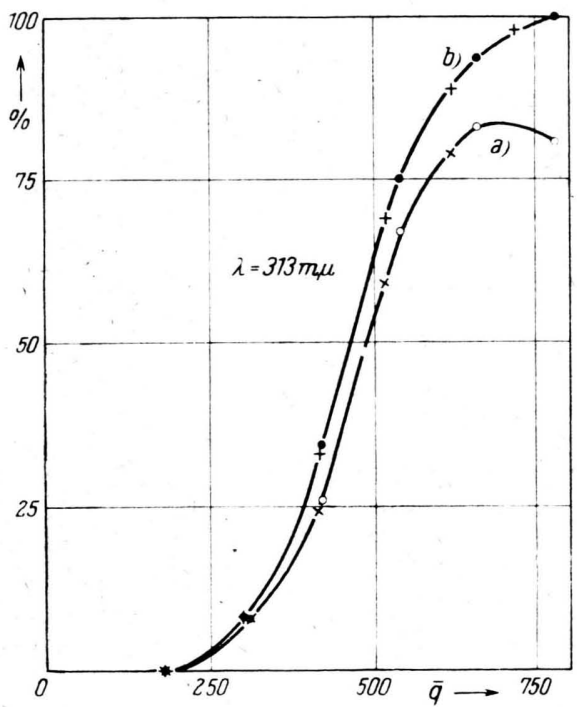

Abb. 10. Umwandlung von T.F. in Alkohol zu PhotoT.T.C. durch Licht der Wellenlänge $\lambda=313 \mathrm{~m} \mu$, berechnet a): aus den Kurven a Abb. 9; b): aus den Kurven b Abb. 9. Abszisse: mittlere absorbierte Quantenzahl pro Molekül $\bar{q}$. Ordinate: Umwandlung in $\%$.

die sich aus der Änderung von $\varkappa_{265}$ ergeben, also den Ablauf der Erscheinungsform a), während Abb. 12 den Ablauf der Erscheinungsform b) darstellt. Außer- 


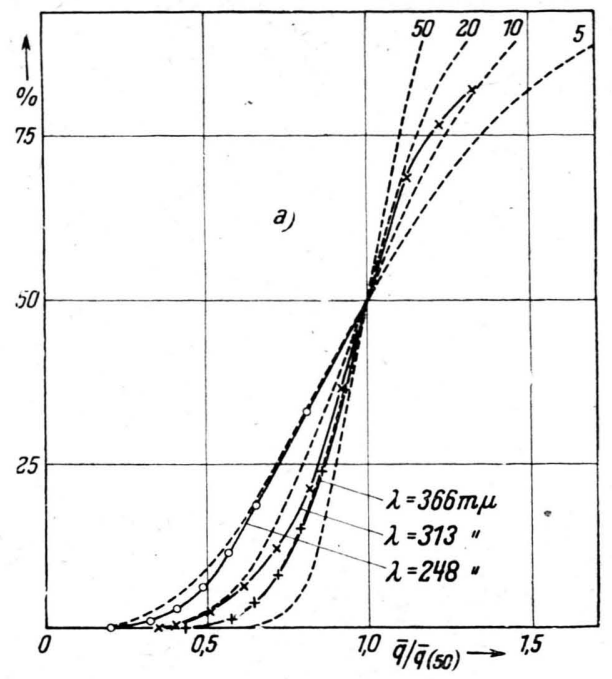

Abb. 11. Dosis-Effekt-Kurve der Umwandlung von T.F. zu Photo-T.T.C. durch Licht der Wellenlänge $\lambda=248$, 313 und $366 \mathrm{~m} \mu$, berechnet aus der Änderung von $\varkappa_{265}$. Zum Vergleich, gestrichelt, theoretische Dosis-Effekt-Kurven von Mehrtreffervorgängen für $n=5,10,20$, 50 Treffer. Abszisse: mittlere absorbierte Quantenzahl $\bar{q}$, dividiert durch die für $50 \%$ Umwandlung erforderliche

Quantenzahl $\overline{q_{50}}$. Ordinate: Umwandlung in $\%$.

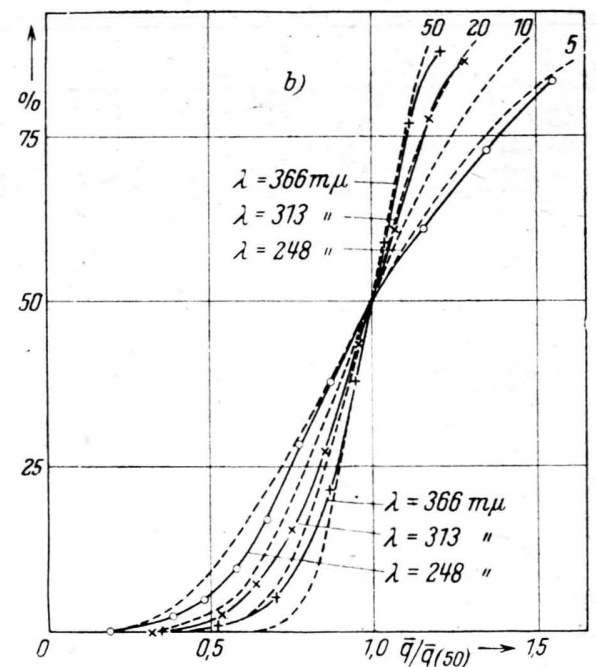

Abb. 12. Dosis-Effekt-Kurve der Umwandlung von T.F. zu Photo-T.T.C. durch Licht der Wellenlänge $\lambda=248$, 313 und $366 \mathrm{~m} \mu$, berechnet aus der Änderung von $\varkappa_{490}$. Zum Vergleich, gestrichelt, theoretische Dosis-Effekt-Kurven von Mehrtreffervorgängen für $n=5,10,20$, 50 Treffer. Abszisse: mittlere absorbierte Quantenzahl $\bar{q}$, dividiert durch die für $50 \%$ Umwandlung erforderliche

Quantenzahl $\overline{q_{50}}$. Ordinate: Umwandlung in $\%$.

dem sind in Abb. 11 und 12 die theoretischen Mehrtrefferkurven für einen 5-, 10-, 20- und 50-Treffervorgang gestrichelt eingezeichnet..
Im Gegensatz zu den beiden zuerst beschriebenen Umwandlungen stellt sich die Umwandlung von T.F. (II) in das Photoprodukt (III) nach der Form seiner Dosis-Effekt-Kurve als typischer Mehrtreffervorgang dar. Sowohl die Umwandlungskurven als Funktion der pro Molekül absorbierten mittleren Quantenzahl $\bar{q}$ als auch die Dosis-Effekt-Kurven zeigen die charakteristische S-Form mit waagerechter Tangente an die Kurven im Koordinatenanfangspunkt.

Die Entfärbung der anfänglich roten Lösung und die am Ansteigen von $\varkappa_{265}$ erkennbare Bildung von Photo-T.T.C. erfolgen beide weitgehend parallel in den gleichen Zeiträumen. Die Entfärbung tritt zu $100 \%$ ein, die Bildung des Photoprodukts im günstigsten Fall zu 92\%. Bei weiterer Bestrahlung wird das Photoprodukt, das selbst auch lichtempfindlich ist, langsam wieder zerstört. Dieser Zerstörungseffekt überlagert sich der Bildung von Photo-T.T.C. und verfälscht die Umwandlungskurven, allerdings erst merklich bei Umwandlungen über $50 \%$. Außer der Form der Umwandlungskurven, die aus der Änderung von $\varkappa_{265}$ und $\varkappa_{490}$ berechnet sind, ist auch deren Quantenbedarf und seine Wellenlängenabhängigkeit einander sehr ähnlich. In der im folgenden treffertheoretisch durchgeführten Berechnung der Quantenbilanz sind die aus dem Anstieg von $\varkappa_{265}$ berechneten Umwandlungskurven zugrunde gelegt, da sie als die eigentliche Erscheinungsform der Bildung von PhotoT.T.C. angesehen werden müssen und einen Vergleich mit der Umwandlung von T.T.C. zum Photoprodukt gestatten.

Die Resultate für die 3 verschiedenen Wellenlängen, mit denen die Umwandlung durchgeführt wurde, zeigt Tab. 1. Eine systematische Abhängigkeit von der Intensität des eingestrahlten Lichtes konnte in dem untersuchten Bereich nicht gefunden werden. Die Größe des formalen Treffbereichs $v$ ist in der oben dargestellten Weise berechnet. Eine gewisse Unsicherheit liegt in der graphischen Ermittlung der Trefferzahl $n$ aus den Dosis-Effekt-Kurven, da sich die experimentell gefundenen Kurven in Abb. 11 und 12 nicht genau mit einer theoretischen Kurve decken. Ferner kann bei Mehrtreffer-Vorgängen, die in mehreren Schritten erfolgen, der formale Treffbereich $v$ für die einzelnen Schritte verschiedene Werte haben; der errechnete formale Treffbereich $v$ stellt dann nur einen Mittelwert dar. Darin liegt ein weiterer Unsicherheitsfaktor für die Ermittlung von $n$ und $v$. Die Trefferzahlen $n$ und die daraus berechneten formalen Treffbereiche $v$ sind deshalb nur als Annäherungswerte zu betrachten. 


\begin{tabular}{|c|c|c|c|c|}
\hline 1 & 2 & 3 & 4 & 5 \\
\hline $\begin{array}{l}\text { Eingestrahlte } \\
\text { Wellenlänge } \lambda \\
\qquad \mathrm{m} \mu\end{array}$ & $\begin{array}{l}\text { Bei } 50 \% \text { Umwand- } \\
\text { lung im Mittel pro } \\
\text { Molekül absorbierte } \\
\text { Quantenzahl } \\
\bar{q}_{(50) \text {, exp. }}\end{array}$ & $\begin{array}{c}\text { Anzahl der zur Er- } \\
\text { zielung des Effekts } \\
1 \text { erforderliche } \\
\text { Trefferzahl } \\
n\end{array}$ & $\begin{array}{c}\text { Aus Trefferzahl } n \\
\text { für } 50 \% \text { Effekt } \\
\text { theoretisch berech- } \\
\text { nete aktive Quanten- } \\
\quad \text { zahl } \\
\bar{q}_{(50), \text { theor. }}\end{array}$ & $\begin{array}{c}\begin{array}{c}\text { Formaler } \\
\text { Treffbereich }\end{array} \\
\text { Molekülvolumen } \\
=v / V\end{array}$ \\
\hline $\begin{array}{l}248 \\
313 \\
366\end{array}$ & $\begin{array}{l}125 \\
490 \\
350\end{array}$ & $\begin{array}{c}6 \\
15-20 \\
20-25\end{array}$ & $\begin{array}{l}5,6 \\
17 \\
22\end{array}$ & $\begin{array}{l}1 / 22 \\
1 / 30 \\
1 / 16\end{array}$ \\
\hline
\end{tabular}

Tab. 1.

Die Bildung des Photoproduktes aus T.F. steht, wie oben ausgeführt, vom Standpunkt der Quantenbilanz in einem engen, zwangsläufigen Zusammenhang mit der. Entfärbung des Moleküls. Auch vom chemischen Standpunkt ist das gut zu verstehen. T.F. geht in das Photoprodukt durch zwei Ringschlüsse über (vgl. Form II und III), von denen z. B. der Ringschluß zwischen den beiden Stickstoffatomen zu einem Fünfer-Ring für sich allein bereits eine Entfärbung der Lösung bedeuten würde, wie man am Beispiel des T.T.C. sieht. Ferner zeigt ein Vergleich der Formeln von T.T.C. (I) und T.F. (II) mit der des Photoprodukts (III), daß der Übergang von II zu III für die Bildung zweier Ringschlüsse unter Abtrennung von 3 Wasserstoffatomen einen größeren Quantenbedarf erfordern muß als der Übergang von I zu III, bei dem nur ein Ringschluß unter Abtrennung von 2 Wasserstoffatomen erfolgt.

Das auf photochemischem Wege dargestellte Photoprodukt (III) entsteht, ausgehend von T.T.C. (I) oder von T.F. (II), in der Lösung als das Kation 5-Phenyl2.3-diphenylen-tetrazoliumchlorid ${ }^{1}$. In bezug auf ein zugehöriges Anion entstanden keine Schwierigkeiten, sofern T.T.C. das Ausgangsmaterial war, da dessen Anion als $\mathrm{Cl}^{-}$in Lösung verblieben war. Bei der Bildung des Kations aus T.F. ist zunächst nicht unmittelbar ersichtlich, wie ein zugehöriges Anion entstehen kann. Bei der Beurteilung des gemessenen, erforderlichen Quantenbedarfs ist daher vom chemischen Standpunkt zu berücksichtigen, ob vielleicht ein Teil der Quanten zur Bildung des Anions verbraucht wird. Es ist auch noch die Möglichkeit zu erwägen, ob sich aus T.F. primär wirklich das Anion bildet oder das der Struktur nach mit ihm identische aber ungeladene Radikal. Nach einem Vorschlag von Hrn. Prof. R. K u h n wurde daher der Quantenbedarf auch in einer Lösung gemessen, die das fehlende Anion als $\mathrm{NO}_{3}^{-}$(in Form des leicht dissoziierenden Silbernitrats) in der Lösung in gleicher Konzentration wie das T.F. zugesetzt enthielt. Dabei verwandelte sich das T.F. durch den Zusatz von Silbernitrat bereits im Dunkeln in einigen Stunden in einen Stoff, dessen Absorptionsspektrum mit dem des T.T.C. übereinstimmt und dessen photochemische Umwandlung auch ganz wie diejenige des T.T.C. verläuft, also als typischer Eintreffervorgang.

Weiterhin wurde der alkoholischen Lösung von T.F. das Anion als $\mathrm{Cl}^{-}$in Form von $\mathrm{HCl}$ gleicher Konzentration hinzugesetzt, um den Bedingungen einer alkoholischen Lösung von T.T.C. möglichst nahe zu kommen. Der Zusatz von $\mathrm{HCl}$ verursachte keine chemische Veränderung der T.F.-Lösung, das Ab-

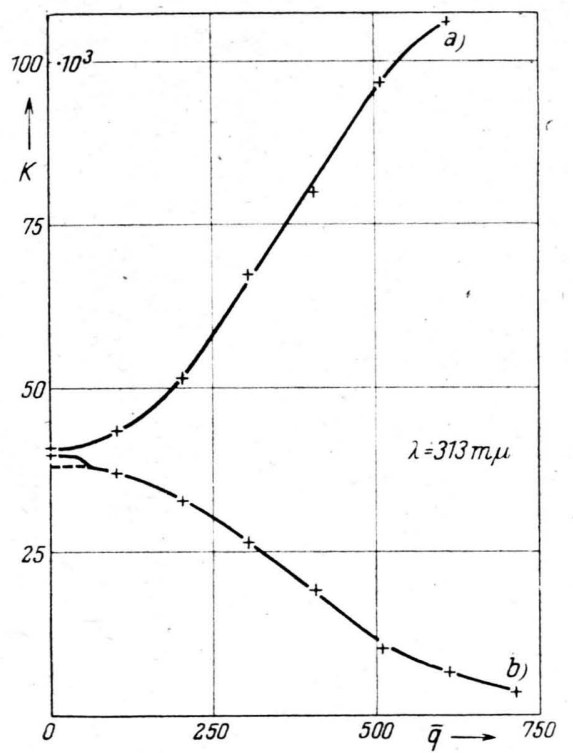

Abb. 13. Änderung des Absorptionskoeffizienten $\%$ von T.F. in Alkohol bei Zusatz von $\mathrm{HCl}$ durch Absorption von Licht der Wellenlänge $\lambda=313 \mathrm{~m} \mu$ bei den Meßwellenlängen, Kurve a: $\lambda=265 \mathrm{~m} \mu$; Kurve b: $\lambda=490 \mathrm{~m} \mu$. Abszisse: mittlere absorbierte Quantenzahl pro Molekül $\bar{q}$. Ordinate: molarer Absorptionskoeffizient $\varkappa$. 


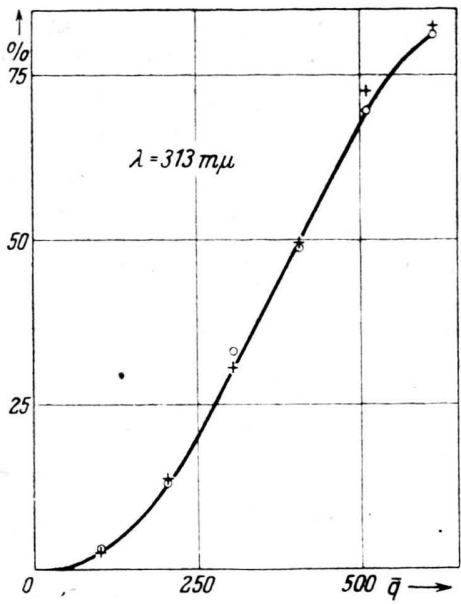

Abb. 14. Umwandlung von T.F. in Alkohol bei Zusatz von $\mathrm{HCl} \mathrm{zu}$ Photo-T.T.C. durch Licht der Wellenlänge $\lambda=313 \mathrm{~m} \mu$, berechnet aus Kurve a Abb. 13. Abszisse: mittlere absorbierte Quantenzahl pro Molekül $\bar{q}$. Ordinate: Umwandlung in \%.

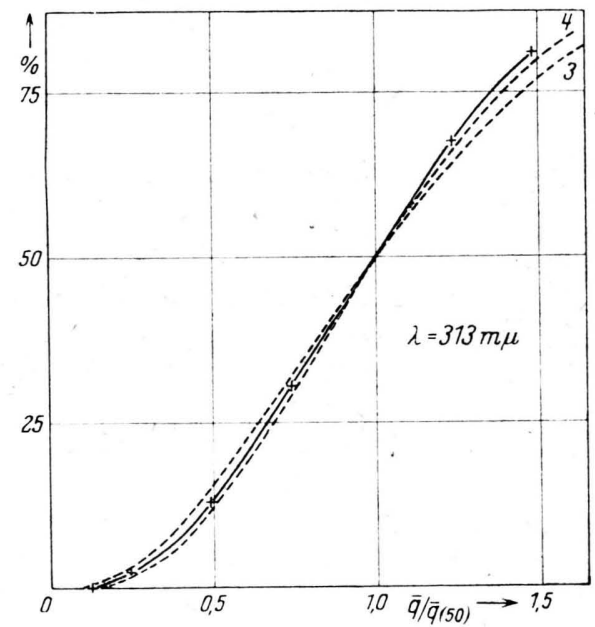

Abb. 15. Dosis-Effekt-Kurve der Umwandlung von T.F. in Photo-T.T.C. bei Zusatz von $\mathrm{HCl}$ durch Licht der Wellenlänge $\lambda=313 \mathrm{~m} \mu$, berechnet aus der Umwandlungskurve Abb. 14. Zum Vergleich, gestrichelt, theoretische Dosis-Effekt-Kurven von Mehrtreffervorgängen für $n=3$ und 4 Treffer. Abszisse: mittlere absorbierte Quantenzahl $\bar{q}$, dividiert durch die für $50 \%$ Umwandlung erforder-

liche Quantenzahl $\bar{q}_{50}$. Ordinate: Umwandlung in $\%$.

sorptionsspektrum blieb dasselbe. Bei der Bestrahlung ergaben sich dagegen wesentliche Unterschiede gegenüber der reinen T.F.-Lösung. In Abb. 13 ist die Änderung der Absorptionskoeffizienten $\varkappa_{265}$ und $\varkappa_{490}$ infolge der Einstrahlung monochromatischen Lichtes der Wellenlänge $313 \mathrm{~m} \mu$ dargestellt. Abb. 14 zeigt die daraus berechneten Umwandlungskurven und
Abb. 15 die dazugehörigen Dosis-Effekt-Kurven. Die zu der Umwandlung eines Moleküls erforderliche Trefferzahl $n$ beträgt etwa 4 . Die bei $50 \%$ Umwandlung im Mittel pro Molekül zur Wirkung gelangende Quantenzahl errechnet sich danach zu $q_{\text {akt, } 50}=3,6$. Die bei demselben Umwandlungsprozentsatz im Mittel pro Molekül absorbierte Quantenzahl $q_{\text {abs,50 }}$ beträgt aber etwa 400 , woraus sich der formale Treffbereich errechnet zu $v / V=1 / 110$. Bei Einstrahlung von Licht der Wellenlänge $\lambda=248 \mathrm{~m} \mu$ wurde ebenfalls $n$ etwa gleich 4 gefunden. Die bei $50 \%$ Umwandlung im Mittel pro Molekül absorbierte Quantenzahl beträgt jedoch 120 , woraus sich der formale Treffbereich errechnet zu $v / V=1 / 33$.

Die Meßresultate an alkoholischen Lösungen von T.F. mit und ohne Zusatz von $\mathrm{HCl}$ lassen sich folgendermaßen zusammenfassen:

a) Für den eigentlich untersuchten Hauptprozeß, die Umwandlung von T.F. zu Photo-T.T.C. werden etwa 4 Quanten pro Molekül verbraucht.

b) Ist nur T.F. in alkoholischer Lösung vorhanden, so ist dieser Prozeß nur gleichzeitig mit anderen, sekundären Prozessen möglich, von denen man vermuten kann, daß sie der Bildung des negativen Ions dienen.

c) Die durch diesen Sekundärprozeß bedingte Steigerung der zur Erzielung eines Effekts notwendigen Trefferzahl $n$ ist abhängig von der Energie des eingestrahlten Quants; bei der energiereichsten Wellenlänge, $\lambda=248 \mathrm{~m} \mu$, sind insgesamt etwa $n=6$ Treffer, bei den energieärmeren Wellenlängen, $\lambda=$ 313 und $\lambda=366 \mathrm{~m} \mu$, etwa $15-25$ Treffer notwendig.

d) Die Anzahl der pro Molekül absorbierten Quanten übertrifft die Anzahl der für den Hauptprozeß notwendigen Treffer (4) um ein Vielfaches, was sich in der geringen Größe des formalen Treffbereichs $v$ ausdrückt. Wie schon erwähnt, könnte die geringe Größe des formalen Treffbereichs bzw. der Wirkungswahrscheinlichkeit $p$ dadurch bedingt sein, daß die einzelnen Teile des Moleküls im Moment der Absorption eine bestimmte, für die Bildung des Photoproduktes günstige Lage haben müssen. Denn bei T.F. sind auf Grund von cis-trans-Isomerien der beiden Doppelbindungen und der mehr oder weniger eingeschränkten freien Drehbarkeit der Einfachbindungen eine Reihe verschiedener Konstellationen der Teile des Moleküls zueinander möglich.

Die dargestellten definierten photochemischen Prozesse an isolierten Molekülen in Lösung haben zu folgenden Ergebnissen geführt, die unter den ge- 
machten Voraussetzungen allgemeine Gültigkeit haben.

1. Bei der Untersuchung der Quantenbilanz einer photochemischen Reaktion muß man klar unterscheiden zwischen dem Begriff des „absorbierten“ Quants und demjenigen des für die untersuchte Reaktion ,,aktiv“ wirkenden Quants.

2. Es wird ein Weg gezeigt, wie man die Anzahl der ,aktiven“ Quanten mit Hilfe der Treffertheorie bestimmen kann. Der Quotient aus „aktiven“ und ,,absorbierten“ Quanten ist immer kleiner oder höchstens gleich eins, weil naturgemäß nur ,absorbierte“ Quanten ,aktiv“ sein können, $\bar{q}_{\mathrm{akt}} / \bar{q}_{\mathrm{abs}} \leqq 1$. Die Energie derjenigen absorbierten Quanten $\bar{q}_{\mathrm{abs}}-\bar{q}_{\mathrm{akt}}$. die nicht ,,aktiv“ sind, wird für andere Vorgänge wie z. B. Fluoreszenzerregung oder Schwingungsanregung im Molekül verbraucht, die mit dem untersuchten Prozeß keinen unmittelbaren Zusammenhang haben.

3. Zu einer vollständigen Bestimmung der Quantenbilanz einer photochemischen Reaktion gehört demnach erstens die Angabe der Quantenempfindlichkeit $\gamma^{12,13}$

$$
\gamma=\frac{\text { Anzahl der umgewandelten Moleküle }}{\text { Anzahl der absorbierten Quanten }}
$$

und zweitens die Angabe, welcher Bruchteil der „absorbierten“ Quanten für den untersuchten Prozeß „,aktiv“ zur Wirkung gelangt, d. h. die Angabe des Quotienten $q_{\text {akt }} / \bar{q}_{\text {abs }}$.

4. Bei Gültigkeit des Quantenäquivalentgesetzes von $\mathrm{E}$ in s te i $\mathrm{n}^{\mathbf{1 4}}$ ist zu erwarten, daß $\gamma=1$ ist. Die besonders in der organischen Photochemie sehr häufi-

12 E. Warburg u. W. Rump, Z. Physik 47, 305 [1928].

${ }^{13}$ K. F. B o nho effer u. P. H a r te ck, Grundlagen der Photochemie, Th. Steinkopff, Leipzig 1933.

${ }_{14}$ A. Einstein, Ann. Physik 17, 132 [1905]. gen Abweichungen von eins wurden dadurch erklärt, daß der beobachtbare Vorgang ein Sekundärprozeß ist. Nach den hier dargelegten Ergebnissen können Abweichungen jedoch, auch bei einfachen Primärprozessen, dadurch eintreten, daß nicht alle ,absorbierten "Quanten für den betreffenden Prozeß ,,aktiv" sind, also $\bar{q}_{\text {akt }} / \bar{q}_{\text {abs }}<1$ ist.

5. Die Ergebnisse lassen sich noch in anderer Form darstellen, wenn man in Analogie zur Quantenempfindlichkeit $\gamma$ eine neue Quantenempindlichkeit $\gamma^{*}$ einführt, bei der an Stelle der absorbierten Quanten die aktiven treten, also

$$
\gamma^{*}=\frac{\text { Anzahl der umgewandelten Moleküle }}{\text { Anzahl der aktiven Quanten }} .
$$

6. Die Angabe von $\gamma$ und $\gamma^{*}$ liefert ebenfalls eine vollständige und oft bequeme Aussage über die Energiebilanz einer photochemischen Reaktion. In der Definition von $\gamma^{*}$ sind diejenigen Quanten, die für die untersuchte Wirkung verlorengehen, nicht enthalten; $\gamma^{*}$ gibt daher den unmittelbaren Zusammenhang zwischen dem einzelnen Quant und der durch seine Energie hervorgerufenen Reaktion wieder. Die Anzahl $n$ der ,aktiven“ Quanten, die im einzelnen Molekül zu dem untersuchten Prozeß beitragen, ist gleich dem reziproken Wert $1 / \gamma^{*}$. Der Bruchteil der „absorbierten“ Quanten, die „aktiv“ sind, ist schließlich gleich dem Quotienten aus $\gamma$ und $\gamma^{*}$, $\bar{q}_{\mathrm{akt}} / \bar{q}_{\mathrm{abs}}=\gamma / \gamma^{*}$.

Meiner lieben Mutter, Frau Is olde $\mathrm{Hau}$ aer, danke ich herzlich für die Anregung zu dieser Arbeit und für stete Förderung bei der Ausführung derselben. Ferner danke ich auch meinem hochverehrten Lehrer, Hrn. Prof. Dr. W. Koss el, für das Interesse, das er der Arbeit während ihres Entstehens und in eingehenden Besprechungen entgegengebracht hat. 